\title{
CARACTERIZACIÓN DE SISTEMAS DE PRODUCCIÓN LECHERA DE ECUADOR
}

\author{
CHARACTERIZATION OF MILK PRODUCTION SYSTEMS IN ECUADOR \\ Narcisa Requelme y Nancy Bonifaz
}

Centro de Investigación de la Leche CILEC, Universidad Politécnica Salesiana del Ecuador, Cayambe, Av. Natalia Jarrin y 9 de Octubre, Telf. (593 2) 3962885, Ecuador

Autor para correspondencia: nrequelme@ups.edu.ec

Manuscrito recibido el 12 de marzo de 2012. Aceptado, tras revisión, el 24 de mayo de 2012.

\begin{abstract}
Resumen
Esta investigación generó información actualizada de los Sistemas de Producción Lechera (SPL) en Ecuador en función de sus regiones agroclimáticas con el objetivo de identificar limitaciones, potencialidades e interacciones que determinen su productividad y sostenibilidad. Se muestrearon Unidades Productivas (UPA's) de manera aleatoria bajo un esquema de muestreo bietápico, estratificado de acuerdo al tamaño de finca para luego proceder por cuotas en siete provincias de la Sierra y Costa. Se determinaron las siguientes variables por SPL: número de integrantes de en las familia, nivel de instrucción, edad, sistema de riego, tipo de hato, cruce de ganado, razas y mezclas genéticas, superficie destinada para pasto, tipo de alimentación del ganado, precios y comercialización de los productos. Los SPL investigados dan cuenta de la diversidad de UPA's que existen en el país, desarrollándose desde pequeñas extensiones como una forma de vida campesina con una economía de sobrevivencia hasta las de grandes extensiones en las que se ha tecnificado la producción y se obtienen altos niveles de rentabilidad, con reinversión en la actividad. Los criterios técnicos que manejan los productores también son diversos y dependen de la posibilidad económica para implementarlos, del conocimiento que se tiene sobre ellos y de los incentivos en el precio de la leche que pueden motivar su mejora.
\end{abstract}

Palabras claves: sistemas de producción, lechería, Ecuador

\begin{abstract}
This work is the research on milk production systems (SPL) considering agro-climatic regions to identify limitations, potentials and interactions that could help to determine productivity and sustainability. The study uses an aleatory sample of production unities (UPA's) then a classification was proposed in two steps. First, a study depending on the size of the farm, then a study where the variables are the number of individuals of the family, instruction level, age, irrigation systems, cattle quantity, cattle species, cattle feed, pasture type, pasture area, price and marketing of the products.

The investigated SPL shown a broad variety of UPA's, the smaller ones associated with rural economy and low profit benefits and the bigger ones associated with large area of technical production with high profit benefits allowing re-investments in the activity. The main factor that determines the technical criteria of the milk produces is the milk price, which fluctuations in the market are decisive in the industrialization of the milk production.
\end{abstract}

Keywords: production systems, milk industry, Ecuador.

Forma sugerida de citar: Requelme, N. y N. Bonifaz. 2012. Caracterización de sistemas de producción lechera de Ecuador. La Granja. Vol. 15(1): 55-69. ISSN: 1390-3799. 


\section{Introducción}

A nivel mundial el tema de Sistemas de Producción Lechera (SPL) ha sido tema de preocupación de diferentes estamentos como la Organización de las $\mathrm{Na}$ cione Unidad para la Alimentación y la Agricultura (FAO) que enlaza el tema con la pobreza y tiene como preocupación principal el cómo mejorar los medios de subsistencia de los pequeños agricultores en un mundo cambiante.

Se puede definir a un sistema como un grupo de componentes que pueden funcionar recíprocamente para lograr un propósito común. Son capaces de reaccionar juntos al ser estimulados por influencias externas. El sistema no está afectado por sus propios egresos y tiene límites específicos en base de todos los mecanismos de retroalimentación significativos" (Spedding, 1979).

Muchos autores consideran que en la investigación agropecuaria, el enfoque sistémico fue introducido como consecuencia de la "revolución verde", en el ámbito de los pequeños productores, porque los "paquetes tecnológicos" desarrollados en universidades y centros de investigación no lograron responder a los problemas de ese universo.

Así, en el país se han realizado varias investigaciones y se han desarrollado proyectos puntuales como el Proyecto de Análisis y mejoramiento de Sistemas de Producción (MAGAP-GTZ., 1994) que dejó bases metodológicas, resultados y recomendaciones pero que lamentablemente han sido acogidas por pocas instituciones y profesionales. El Instituto Nacional Autónomo de Investigación Agropecuaria (INIAP), a finales de los años setenta, desarrolló un proyecto de investigación aplicado en sistemas de producción de leche. Se desarrollaron modelos de simulación sobre los factores que afectan los sistemas de producción de pequeños productores de la provincia del Carchi y otras investigaciones sobre sistemas de producción mixtos que se desarrollaron se han desarrollado en las provincias de Chimborazo y Cañar.

El fomento de las actividades productivas precisa de información fehaciente de la realidad del sector, pero la dispersión o falta de información y conocimiento, no permite construir políticas públicas que solucionen la problemática, esta situación afecta no solo al Estado como tal sino también a las instituciones que realizan acciones de investigación, formación y capacitación, tanto de profesionales como de productores agropecuarios, debido a que no se establecen claramente las demandas así como las potencialidades y limitaciones del sector.

Por otro lado, la heterogénea geografía de las cuatro regiones naturales de Ecuador ofrece muy diversos escenarios naturales, climas y microclimas que propician prácticas culturales, variadas y disímiles, en muchos casos para trabajar la tierra. Este sector de la economía, consecuentemente, presenta una caracterización compleja y diversa, cuyo indispensable estudio implica necesariamente un desafío (MAGAP, 2011).

En la investigación se planteó identificar SPL's por áreas agroecológicas para caracterizar técnica y socialmente al sector lechero ecuatoriano, de manera que desde la academia se contribuya al desarrollo de una ganadería sustentable a partir del enfoque de sistemas que permitan entender las dinámicas particulares para mantener el equilibrio de esos sistemas y la obtención de altos niveles de eficiencia, rentabilidad y sostenibilidad. Conocer los sistemas de producción es elemental para construir políticas públicas así como para definir estrategias, acciones de investigación, validación, transferencia de tecnología y capacitación de los productores, tomando en cuenta el entorno ambiental y social.

\section{Materiales y métodos}

\subsection{Población y muestra}

Se revisó información secundaria oficial respecto a los Sistemas de Producción en Ecuador y el referente oficial (Censo Agropecuario del 2001), luego se definieron áreas agroecológicas y socioeconómicas de producción lechera revisando datos de información secundaria: altitud y clima (temperatura y precipitación) reportada por el Instituto Nacional de Meteorología e Hidrología del Ecuador (INAMHI) y el mapa ecológico de Ecuador. En las áreas agroecológicas seleccionadas se identificaron cantones representativos en los cuales la encuesta se aplicó de manera aleatoria bajo un esquema de muestreo bietápico, estratificado de acuerdo al tamaño y, luego, por cuotas para la determinación de las unidades muestrales en las provincias de Carchi, Imbabura, Pichincha, Chimborazo, Azuay, Manabí y Guayas. Una vez que se definió el diseño que se emplearía, se procedió a 
realizar la repartición del tamaño muestral total. Se fijó un nivel de confiabilidad del 95,5\%, con un nivel de error del $3 \%$.

\subsection{Técnicas y herramientas}

Para el levantamiento de la información se visitaron el número de UPA's definido como muestra y se procedió a realizar la encuesta a la vez observación de las unidades productivas y su entorno. Una vez levantada la información y georeferenciadas las UPA's, se procedió a su respectivo procesamiento y el análisis estadístico. A partir de la base de datos de campo se elaboraron mapas temáticos que permitieron visualizar los resultados de manera más didáctica y dinámica.

\section{Zonas agroecológicas de pro- ducción lechera}

Los sistemas que se caracterizan en esta investigación están orientados a la producción lechera y eso define el uso de los recursos así como el tipo de ganado existente en las fincas (Figura 1). Parte de la provincia de Manabí se ubica en la Región Seco Tropical - RST (Chone), al igual que Balzar y el Empalme (Guayas) caracterizada por alturas hasta de 300 msnm y temperatura promedio anual que va desde $\operatorname{los} 23$ a $25^{\circ} \mathrm{C}$, períodos de lluvia que van desde diciembre a mayo y un período seco de junio a noviembre, aunque existen datos de años muy irregulares, en general se presentan períodos secos de entre 5 a 7 meses.

En la Región Húmedo Tropical -RHT se muestreó El Carmen (Manabí) y en Pichincha, lo que corresponde al cantón Quito (Puerto Quito). En esta región existen alturas de 5 a $600 \mathrm{msnm}$ con temperaturas promedio anual de 23 a $25,5^{\circ} \mathrm{C}$, precipitaciones anuales de 2.000 a $3.000 \mathrm{~mm}$, con un período menos lluvioso de diciembre a febrero. Según el mapa bioclimático y ecológico del Ecuador, parte de las provincias de Imbabura (Cotacachi, Ibarra, Urcuqui) y de la provincia del Azuay (Sigsig) pertenecen a la Región Sub Húmedo - Sub Tropical - RSHST que presenta características similares a la RST con la diferencia de que las precipitaciones son mayores y se presentan de manera permanente durante todo el año, la altura va hasta los $2.300 \mathrm{msnm}$ y la tempera- tura promedio anual va desde los 18 a $22^{\circ} \mathrm{C}$, existe una variabilidad de lluvias significativa que no permite identificar claramente las épocas secas y lluviosas.

La zona de Tufiño, Tulcán, Huaca y Julio Andrade; en Imbabura las estribaciones del Cotacachi; una parte de Pintag y Machachi en la provincia de Pichincha; en el Azuay, Paute y Cuenca; y, en Chimborazo los cantones de Riobamba y Chambo pertenecen a la región húmedo temperado - RHTE, la altitud varía entre los 1.800 a $3.000 \mathrm{msnm}$ con temperaturas que van desde los 12 a $18^{\circ} \mathrm{C}$, tanto la época seca como la lluviosa varían considerablemente. En el período de verano se pueden presentar heladas y la neblina se presenta con mucha frecuencia.

A la región muy húmedo sub temperado RMHST corresponden los páramos bajos de Cayambe, en la provincia de Pichincha, Tufiño en la provincia del Carchi, las faldas del Cotopaxi y del Chimborazo; esta región se extiende desde los 3.000 a 4.000 msnm, con temperaturas que van desde los 6 a $12^{\circ} \mathrm{C}$ y precipitaciones que van de 1.000 a $1.500 \mathrm{~mm}$ anuales, en estos últimos años tienen una distribución sin énfasis en algún mes del año (García y Requelme, 2011).

\section{Características de los Sistemas de Producción}

\subsection{El Productor y su familia}

Según el censo de 2001, del $40 \%$ de la población ecuatoriana que reside en el área rural, las dos terceras partes conforman hogares de productores agropecuarios, es decir, que algo más del $25 \%$ de la población ecuatoriana está vinculada a la actividad agropecuaria; ciertamente, el $62 \%$ de la población rural ocupada, trabaja en agricultura. Estas familias tienen entre 3 a 7 miembros que buscan obtener mejores rendimientos y seguir con la actividad tal como lo han venido realizando hasta la actualidad. En la Sierra, la permanencia de los miembros de la familia es común, excepto los hijos/as que estudian y que, generalmente, luego de que logran ser profesionales, no regresan a sus comunidades. La edad de los productores es generalmente superior a los 50 años y podemos decir que se trata de una población adulta con una notable ausencia de jóvenes que releven 
el trabajo de los padres en las fincas, excepto en las UPA's de más de 100 ha en Quito (Puerto Quito) y Pedro Vicente Maldonado en donde la edad de los hombres está entre 27 a 40 años. En lo que se refiere a las mujeres, la edad promedio va de los 33 a los 68 años. Las mujeres en las fincas de la Costa no necesariamente realizan actividades productivas de ganadería sino más bien se dedican a la producción de especies menores y al cultivo de productos para el consumo familiar y de las personas que ayudan o trabajan en la finca. La población femenina más joven (27 a 38 años) se ubica en Quito (Puerto Quito) y las edades mayores de las mujeres se registran en Mejía y Quito (Pintag), en cantones como Cuenca y Paute las mujeres son las que están al frente de la finca.

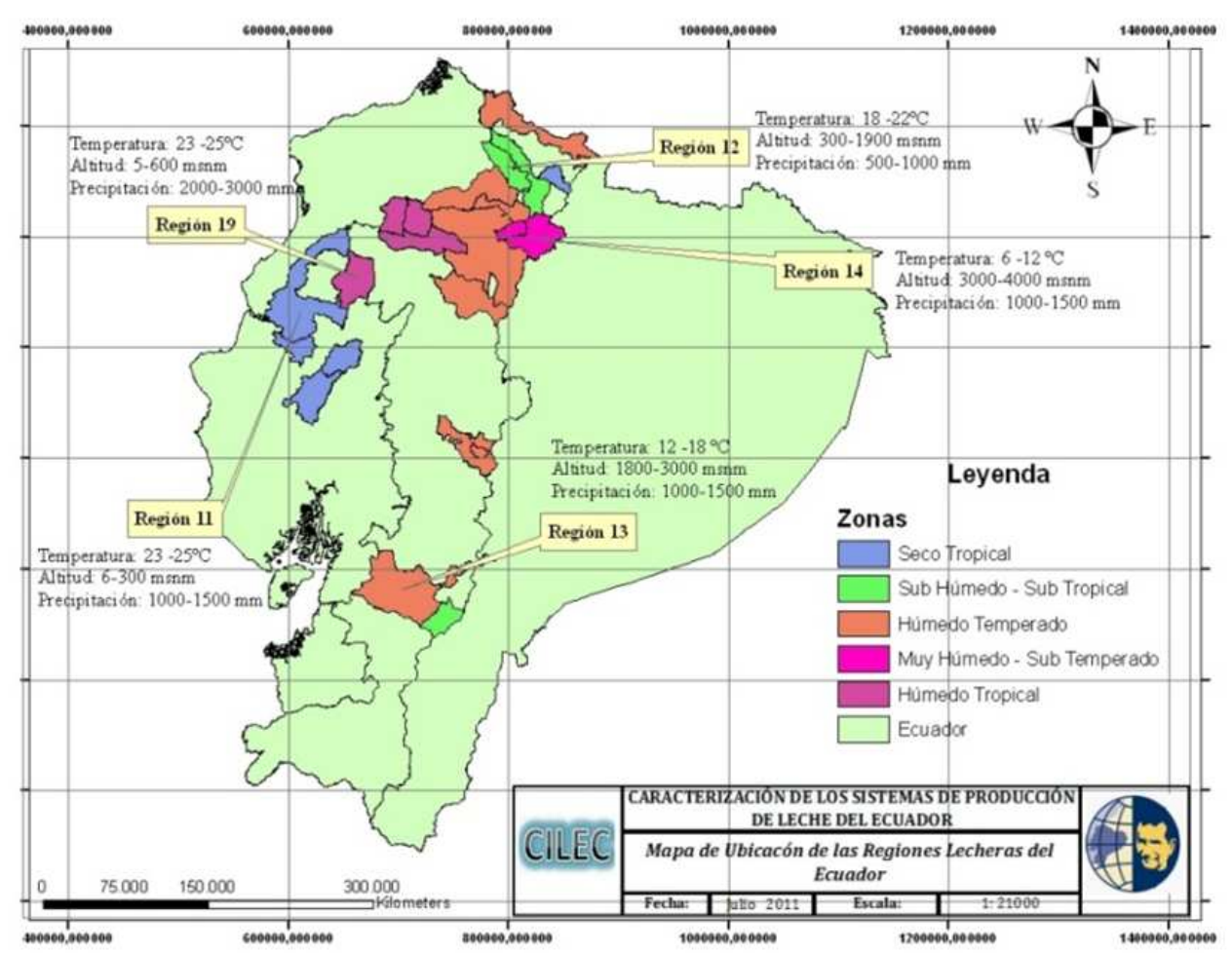

Figura 1. Zonas agroecológicas de producción lechera en Ecuador.

En todos los cantones del 31 al $100 \%$ de productores tienen nivel de educación primario; el 8 al $38 \%$ se sitúa en el nivel secundario, exceptuando los cantones Chambo (50\%), Paute (44\%) y Cayambe $(43 \%)$; y, entre el 12 y $33 \%$ los productores tienen educación superior y generalmente pertenecen a productores de UPA's de mayor superficie: Mejía $(8 \%)$ Riobamba (5\%), Otavalo (3\%), Paute y Cuenca $(8 \%)$, estos productores se dedican completamente a la producción lechera siendo referentes por los índices productivos que obtienen.

En la Costa la mayor parte de los productores se ubican en el estrato 50 a 100 ha y más de 100 ha, mientras que del 50 al $86 \%$ de los productores en cantones de la Sierra, se ubican en los estratos de entre 1 a 5 ha.

\subsection{Límites y componentes de los SPL}

\subsubsection{Tamaño de las UPA's}

El tamaño promedio de las UPA's en la Costa va de 25 a 400 ha y se encontró en la investigaciones propiedades de hasta 1.200 ha. En los otros cantones de la Sierra, en el estrato de 1 a 5 ha el tamaño promedio es de 3 ha; el estrato de 5 a 20 ha presenta tamaños promedio entre 7 a 20 ha; y, en el estrato de más de 20 ha, los valores van desde 20 hasta 120 ha.

LA GRANJA, Revista de ciencias de la vida, 15(1) 2012: 55-69. (C) 2012, Universidad Politécnica Salesiana, Ecuador. 


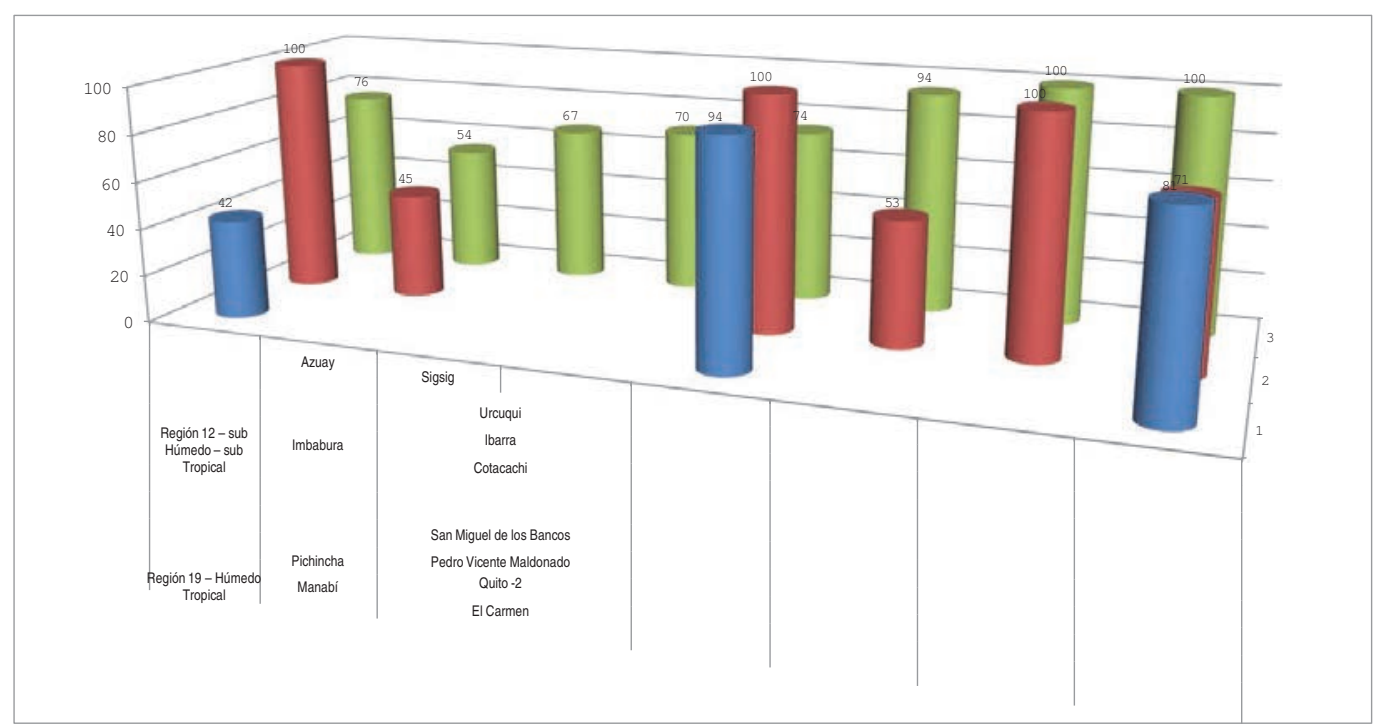

Figura 2. Superficie de UPA's con forraje en UPA's del estrato 3 en las RST y RSHST

Si bien es cierto la superficie de tierra en las fincas de la Costa es grande en su mayoría no disponen de riego, solo entre el 12 al $50 \%$, en el estrato de más de 5 ha y más de 50 ha en la Sierra y en la Costa respectivamente disponen de riego.

Ahora, dentro de las fincas que tienen riego, el tipo, la frecuencia y la cantidad de agua es importante, la mayor parte de los cantones de la Costa tienen riego por inundación en las UPA's menores de 50 ha, mientras que en las de más de 50 ha se tiene riego por aspersión. En cantones de la Sierra se notan mayores superficies de riego por aspersión, especialmente en cantones como Cayambe y Tulcán (ver Figura 3).

\subsubsection{Composición del hato}

En la Costa, la categoría de vacas en producción alcanza un $18 \%$ con un significativo porcentaje de terneras (7 -23\%) y machos (9-18\%) que se crían para la venta. En la Sierra los valores van desde el $18 \%$ hasta el $87 \%$, la categoría de terneras es la siguiente en importancia y los porcentajes van desde el $10 \%$ en el estrato de más de 20 ha en el cantón Quito (Puerto Quito) hasta el 25\% en el estrato de más de 20 ha en Urcuqui, lo cual denota la crianza de hembras de reemplazo. Como en las regiones antes descritas, en la RHTE y RMHSHT, la categoría de vacas en reproducción es la más alta en porcentajes con mínimos de $14 \%$ en el estrato de 5 a 20 ha en Paute (14\%) y hasta $29 \%$ en Quito (Pintag), Chambo y en el estrato de 1 a 5 en Tulcán ( $25 \%)$. En la categoría terneras es notorio los bajos porcentajes de los cantones Riobamba y Mejía (4 a $7 \%$ respectivamente).

\subsection{Ingresos del sistema}

\subsubsection{Producción de forraje}

En la RST, la mayor parte de la superficie de las UPA's se destina a la producción de forraje, siendo menor el porcentaje (39\%) en el estrato de más de 100 ha en los cantones de la Costa, que combinan esta producción con otros cultivos de la zona. En la RHT y RSHT, en todos los estratos de todos los cantones, la mayor parte de la superficie de las UPA's se destinan para la producción de pasto, notándose porcentajes de entre 42 al 54 en el estrato de 1 a 50 ha, de 5 a 20 ha en Quito (Puerto Quito) e Ibarra y de más de 20 ha en Quito (Pintag). En la RHTE y RMHSHT, en el estrato de 1 a 5 ha (excepto en Paute), en el estrato de 5 a 20 ha, la superficie de pasto va desde el $69 \%$ hasta el $100 \%$. Se notan porcentajes menores de superficie de pasto en Cuenca $(33 \%)$, San Pedro de Huaca (42\%) y Tulcán (46\%), en el estrato de más de 20 ha en donde el resto de la superficie se dedica a cultivos destinados para la venta y el autoconsumo como la papa y maíz que son a la vez espacios en los que se realiza rotación de cultivos con pasto (Figura 2). 


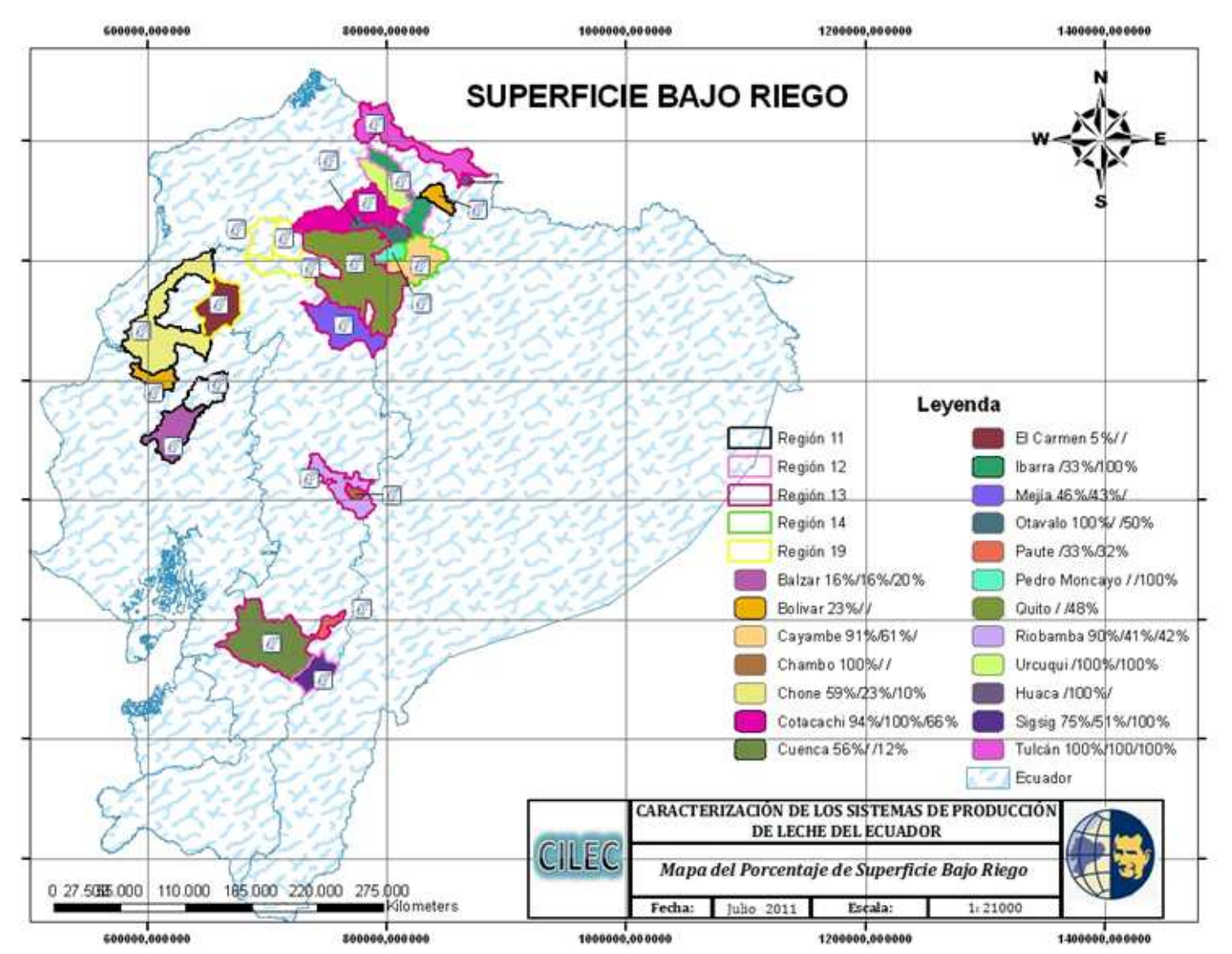

Figura 3. Superficie con riego respecto a superficie total de la UPA por Regiones

\subsubsection{Suplementación}

A más de forrajes, los productores proporcionan otros productos a los animales, especialmente balanceado comercial, en la Costa, por ejemplo, se da de 0,1 a $0,6 \mathrm{~kg} /$ día/animal existiendo fincas grandes en las que no se administra. Es muy común el uso de melaza en épocas de escasez de forraje; en la Costa se da de 2 a $12 \mathrm{~kg} /$ mes en fincas pequeñas y en fincas grandes hasta $15 \mathrm{~kg} /$ mes a vacas en producción. En la Sierra, los valores varían entre 7 hasta $54 \mathrm{~kg} / \mathrm{mes}$. Las sales minerales se suministran en la mayoría de las UPA's de la Costa a las vacas en producción y en pocas fincas a todo el rebaño. Se administra al momento del ordeño y se da a voluntad, en cantidades que oscilan entre 0,01 a 0,4 g/día. En los cantones de la sierra se da entre 0,02 y $0,8 \mathrm{~g} /$ día por día, lo cual de alguna manera está ayudando a que las categorías de vacas y terneras puedan tener una buena alimentación que les ayude a la producción de leche y desarrollo fisiológico.

\subsection{Salidas del sistema}

\subsubsection{Producción de leche}

La producción promedio vaca/día, en el estrato de 1 a 50 ha, en la RST, va de 4,5 a 4,9 1/vaca/día. En el estrato de 50 a 100 ha, el promedio va de 2,7 a 4,2 1/vaca/día y en el estrato de más de 100 ha, el promedio está entre 3 y 5,3 1/vaca/día. En la RHT y RSHST, en los estratos de entre 1 a 50 en la Costa y de 1 a 5 ha en la Sierra se tienen valores de 2,2 a 3,31/vaca/día, valores similares se presentan en el estrato de 50 a 100 ha en la Costa y de 5 a 20 ha en Quito (Puerto Quito) y Pedro Vicente Maldonado. Es significativo el promedio del estrato de 5 a 20 ha en otros cantones de la Sierra que van desde el 8,3 a 14,3 1/vaca día. En el estrato de más de 20 ha la producción va desde 6,5 hasta $15,91 / \mathrm{vaca} /$ día; en cantones que disponen de mejores condiciones de manejo y tecnología como se describe a lo largo de este artículo. En la RHTE y RMHST, en fincas, de 1 
a 5 ha, los valores están entre 3,4 1/vaca/día (Riobamba) hasta 13,5 1/vaca/día (Mejía). En el estrato de 5 a 20 ha, la producción es de 2,9 1/vaca/día en Riobamba hasta 16,1 / vaca/día en Cayambe. En el estrato de más de 20 ha se observan valores de 7,7 a 21, 4 1/vaca/día en los cantones Tulcán y Pedro
Moncayo. En resumen se pude decir que en la Costa el promedio de leche está entre 3,1 y 3,7 1/vaca/día mientras que en la Sierra el promedio está entre 7,9 y 8,6 1/vaca/día, dando un promedio nacional de 5,9 1/vaca/día superior al 4,4 1/vaca/día reportado en el censo del 2001 (ver Figura 4).

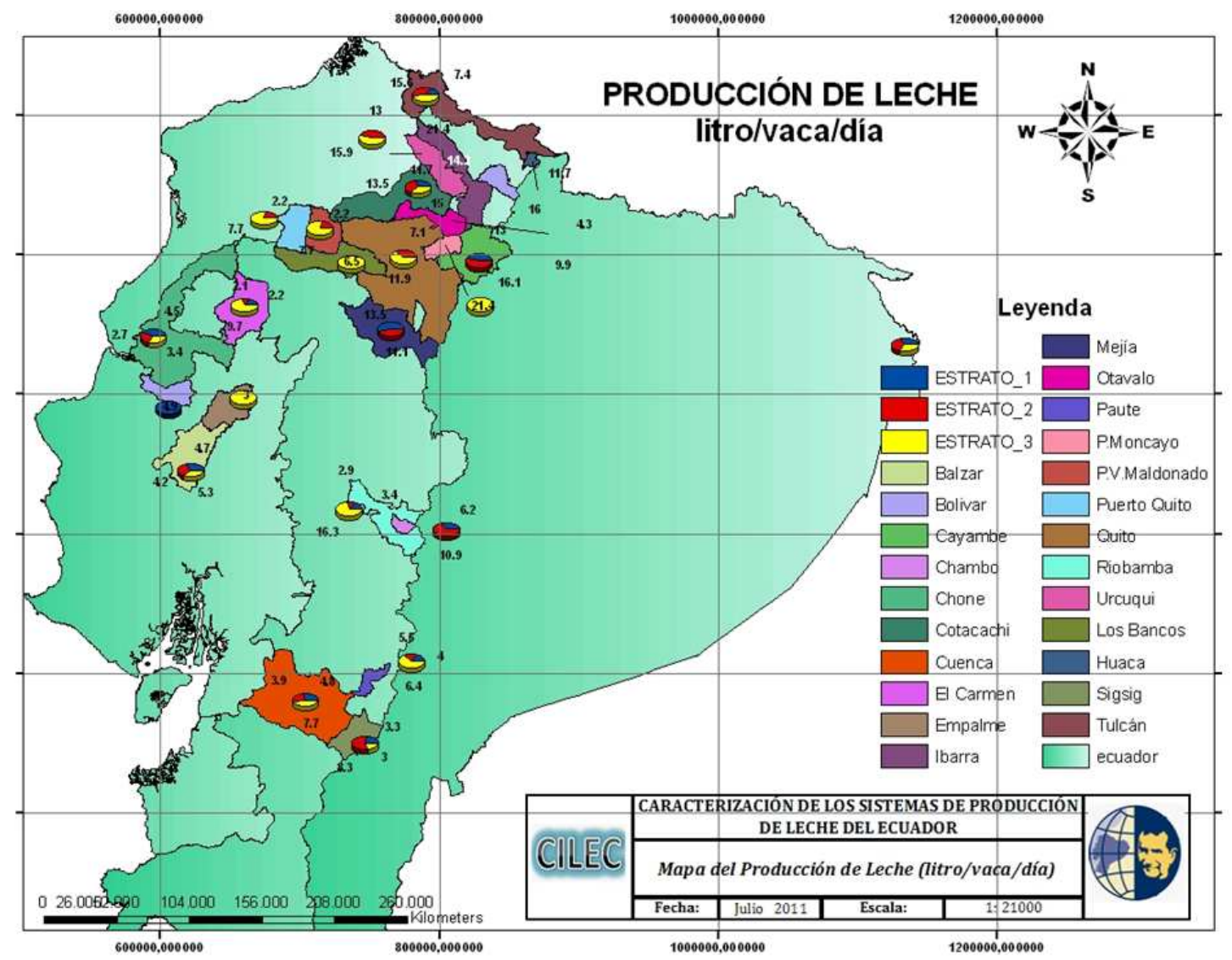

Figura 4. Producción promedio de leche (1/vaca/día) por regiones

\subsubsection{Precio de venta del litro de leche}

Si bien es cierto en el país existe un precio fijado por el Estado de 0,39 USD, los precios que se pagan en la realidad tienen mucho que ver con la calidad que exigen la mayoría de las empresas tanto en la composición física como en las condiciones higiénicas y sanitarias de la leche, excepto en el Austro que no existe mayor control por parte de los compradores y de los productores. Los precios fluctúan de acuer- do a la forma de comercializar; los productores que venden a los centros de acopio reciben un precio entre 0,36 a 0,45 USD. En los cantones de la Sierra, los precios van de 0,32 hasta 0,48 USD siendo siempre mayor en las UPA's de más de 20 ha que van desde 0,37 hasta 0,50 USD (excepto en Chambo: 0,25 USD y Paute: 0,27 USD) que incluyen incentivos de las empresas compradoras a fincas en las que se ha trabajado para la erradicación de tuberculosis y brucelosis especialmente (ver Figura 5). 


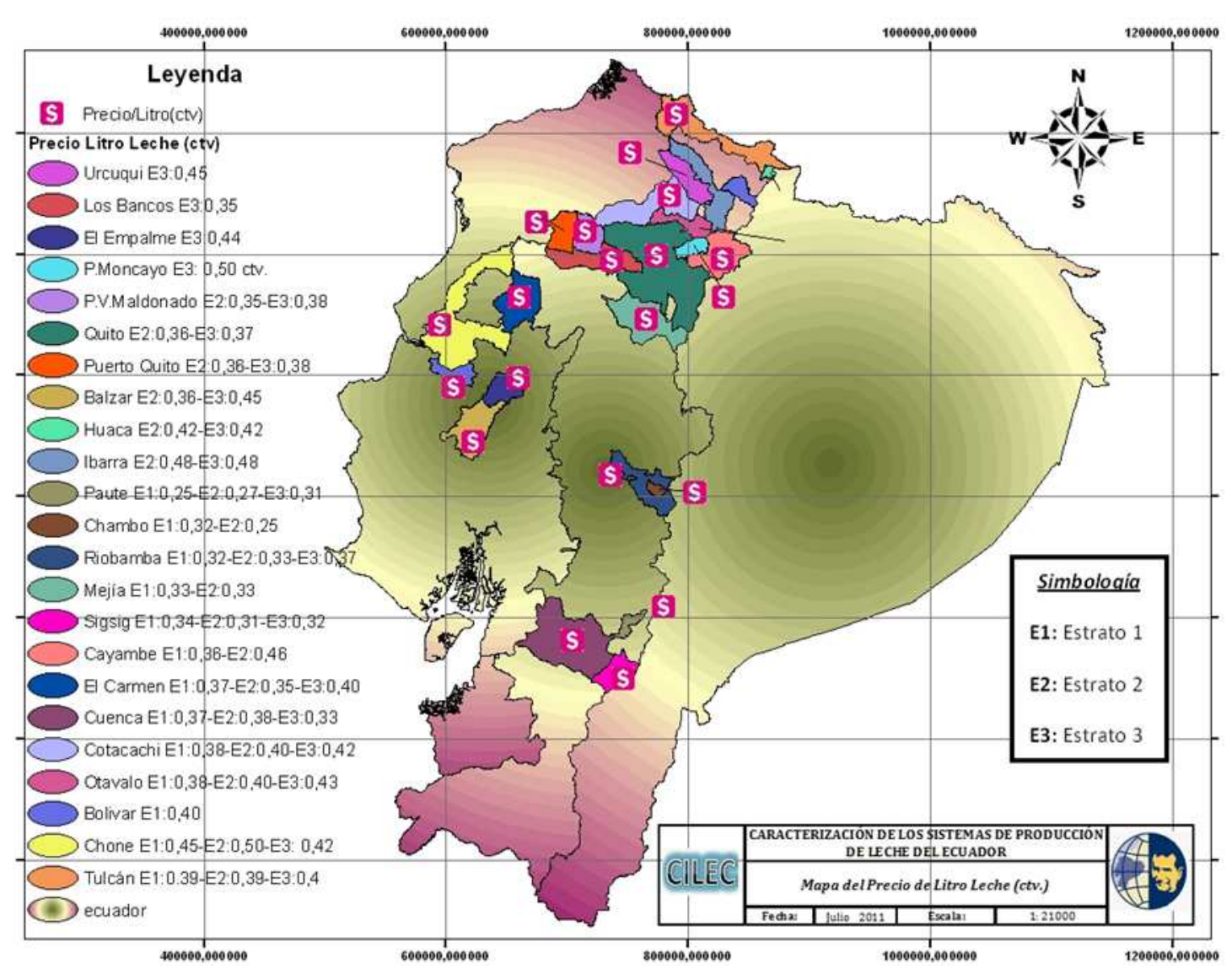

Figura 5. Precio de venta de litro de leche por regiones

\subsubsection{Descarte de animales}

La categoría de vacas de descarte en la RST no se pudo establecer claramente en la investigación, pero por referencia de los productores se conoce que las vacas se mantienen en las fincas hasta que produzcan volúmenes de leche normales, la gente vende ocasionalmente sus vacas para solventar necesidades económicas imprevistas; en algunos cantones de la Costa, las vacas no se reemplazan sino hasta que den por lo menos 5 o 6 crías esto no sucede en las UPA's de más de 20 ha en las cuales los criterios de descarte son los parámetros de producción de leche y la obtención de crías cada año.

En los cantones de la Sierra, especialmente los del estrato de 1 a 5 ha, las vacas son el patrimonio familiar y no es fácil cambiarlas por los gastos que representa la compra de un animal con mejores características de producción. En los estratos de más de 5 ha, el criterio es el mismo pero los producto- res mejoran la calidad de sus vacas reemplazándolas con otras de mejores características que son adquiridas utilizando créditos que se cancelan con la misma producción de leche.

\subsection{Subproductos del sistema}

\subsubsection{Vaconas y vacas vientre}

Si sumamos los porcentajes de las categorías de vaconas y vientres en la RST, vemos que los porcentajes van desde el 15,9 al 39\% en los cantones de la Costa, entendiéndose esta situación por la disponibilidad de superficie para mantener estas categorías que aseguran un reemplazo de vacas de la misma finca, los valores menores están en las UPA's pequeñas $(20 \%)$, teniendo que comprar ganado fuera de la finca con los riesgos sanitarios y otros que ello significa. En los cantones de la Sierra se tiene la restricción del tamaño de las UPA's y los valores van desde 
el 2 al 15\% en Otavalo, Mejía, Tulcán y en el estrato de 5 a 20 ha en los cantones de Chambo (13\%) y Riobamba (17\%).

\subsection{Interacciones principales}

\subsubsection{Carga aninal}

La carga animal en la Costa se encuentra entre 0,17 y 0,69 UBA/ha en las fincas del estrato de 1 a 50 ha; mientras que en el estrato de 50 a 100 ha se tiene una carga animal de 0,98 a 1,16 UBA/ha; y, en el estrato de más de 100 ha se tiene entre 1,2 a 3,13 UBA/ha. Esta carga animal se considera baja considerando que se trata de sistemas extensivos de pastoreo con mayor disponibilidad de forraje (Figura 6).

Los pastizales de Saboya (Panicum maximun) son lo más comunes en los cantones de la Costa, pero se puede también encontrar otras variedades de especies que los productores han introducido, a saber: puntero (Panicum maximun), estrella (Cynodon plectostachum, Cyodon nlenfuensis), maralfalfa (Pennisetum spp.) y otros como el Kingrass (Pennisetum purpureum $x$ Pennisetum typhoides), braquiarias (Brachiaria decumbens) y pasto alemán (Echinochloa polystachia) como los más comunes. Estos pastizales, según Ramírez y Izquierdo (1996), presentan porcentajes de contenido de proteína de $10,4 \%$ en períodos de lluvia y corte a los 60 días; y 7,2\% de proteína en períodos secos y corte a los 78 días. Esto da cuenta, entonces, de un importante aporte de proteínas que permite mantener niveles de producción que representan ingresos suficientes para desarrollar la actividad. Los días de pastoreo varían según se trate de la época de verano o de lluvias así como del tamaño y número de potreros existentes en la finca.

En el caso de las UPA's de la Sierra está entre 0,21 a 0,22 UBA/ha, la carga animal más baja está en el estrato de 1 a 5 de Cuenca $(0,21)$, Tulcán $(0,23)$, Otavalo $(0,24)$, Riobamba y Paute $(0,25)$; el valor más alto en este estrato se logra en Cayambe $(0,54)$; en fincas de 5 a 20 ha, se incrementa, teniéndose valores sobre 0,45 UBA/ha en San Pedro de Huaca hasta llegar a 0,99 en Tulcán, en fincas de más de 20 ha se notan carga animal de 0,77 hasta $1,35 \mathrm{UBA} / \mathrm{ha}$ en Tulcán y Paute.

Estos datos nos llevan a decir que la carga animal es crítica en las UPA's del estrato de 1 a 5 ha, entendiéndose que en estas fincas las prácticas de manejo de potreros casi no existen y que las pasturas son a base de kikuyo (Pennisetum clandestinum), con poco o nada de leguminosas, pero se puede encontrar potreros de holco (Holcus lanatus), grama (Paspalum pigmaeum) ryegrass (Lolium multiflorum), pasto azul (Dactylis glomerata), ryegrass anual (Lolium perenne). En cantones como Cayambe, Mejía y Tulcán se tienen pastizales mejorados a base de pastos introducidos y legumninosas como la alfalfa (Medicago sativa) y trébol blanco (Trifolium repens) en mezclas con diferentes porcentajes de cada uno de ellos o potreros con avena forrajera (Avena sativa) y vicia (Vicia sp.) especialmente en aquellas UPA's en las que se pastorea con cerca eléctrica y se puede manejar exactamente el volumen de pasto que se suministra cada día. Los días de pastoreo varían según se trate de la época de verano o de lluvias así como del tamaño y número de potreros existentes en la finca.

\subsubsection{Estrategias de alimentación}

En las fincas de la Costa no es muy común suministrar concentrados comerciales sino que se usan balanceados elaborados con recursos de la misma. Estos balanceados, por lo general, tienen un porcentaje de proteína que va del 16 al $18 \%$; se da esporádicamente a las vacas en producción y en las UPA's de entre 1 a 100 ha en promedios de 0,10 y 0,70 $\mathrm{kg} / \mathrm{vaca} /$ día. En la Sierra la cantidad varía desde 0,70 a $2 \mathrm{~kg} / \mathrm{vaca} /$ día. Si este suplemento se usa indiscriminadamente tiene repercusiones económicas en las fincas, pero a la vez también puede cambiar el metabolismo de los animales, por cuanto el animal puede ir perdiendo su naturaleza herbívora y acondicionándose solo a aprovechar los carbohidratos solubles del balanceado para la producción de leche. Por otro lado, es importante analizar la cuestión económica ya que por cada $\mathrm{kg}$ de MS consumida como concentrado, el animal reduce $0,5 \mathrm{~kg}$ de forraje, es decir, se está reemplazando el consumo del forraje de menor costo por el de balanceado de mayor costo.

\subsubsection{Estrategias de reproducción}

Los bovinos de raza criolla de origen cebuino son los más comunes en la Costa pero también se presentan cruces de animales criollos con razas tales como Brahman, Charolaise y Holstein. En la Sierra, la mayor parte del ganado se ha mejorado con cruzamientos de criollo con Holstein, Brown Swiss, Jersey 
y Montbeliere principalmente, por lo que se puede decir que la población de animales criollos va disminuyendo. En la Costa, la práctica más general es la monta natural aunque del 10 hasta el $50 \%$ de las UPA's practican la monta natural o la inseminación artificial indistintamente. En las UPA's pequeñas de la Sierra, la monta natural es la práctica general, no así la inseminación artificial que se practica en los estratos de entre 5 a 20 ha y de más de 20 ha, en Paute, Cayambe y Cuenca se practica la monta natural y la inseminación a la vez para lograr la preñez de las vacas y, generalmente, se realiza la monta natural luego de que dos o más inseminaciones no han sido efectivas (Figura 7).

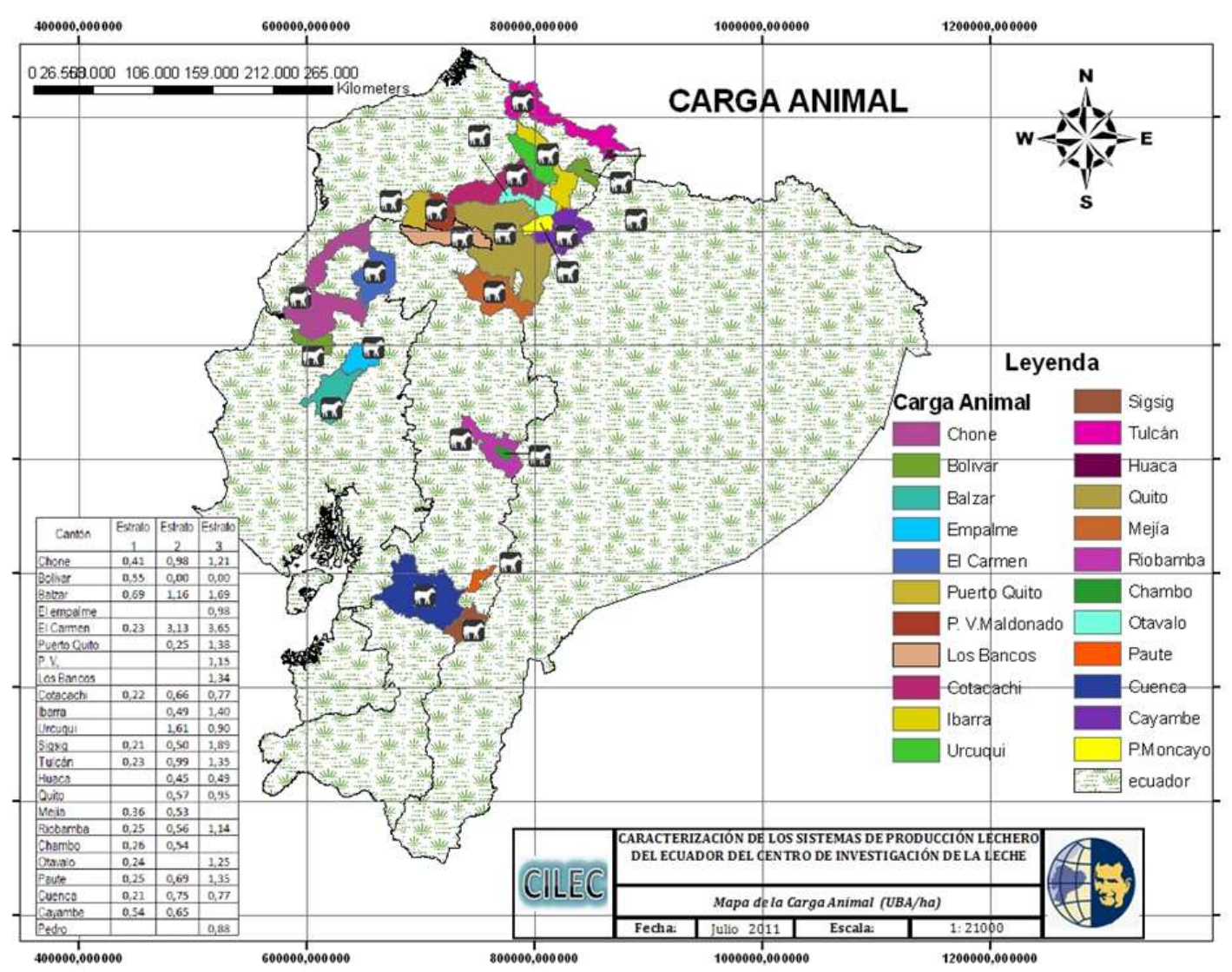

Figura 6. Carga animal por regiones

\subsubsection{Prácticas sanitarias}

La desparasitación es la práctica más común en todo el país; se realiza dos veces al año a todo el ganado y cada seis meses a los terneros; se notan menores porcentajes en Paute (27 y $58 \%$ ) y Tulcán $(58 \%)$ y Cuenca (57\%). La vacunación contra Fiebre Aftosa se realiza en más del $67 \%$ de las fincas en la Costa y entre el 50 a $100 \%$ de las UPA's de todos los cantones de la Sierra (excepto en el estrato de 1 a 5 ha en Sigsig: $92 \%$ ). La vacunación contra brucelo- sis es menor en Riobamba, Mejía, Paute, Quito, Pedro Vicente Maldonado, San Miguel de los Bancos y Tulcán. No se vacuna en Quito (Pintag), Chambo, Otavalo, Paute y Cuenca, existiendo por lo tanto el riesgo de contagio de esta enfermedad tanto a nivel de productores y profesionales que manipulan estos animales así como de consumidores de lácteos provenientes de estas fincas. 


\subsection{Recursos}

\subsubsection{Disponibilidad de infraestructura y equi- pos}

En cuanto a la infraestructura, disponibilidad de maquinaria y equipo se puede decir que los sistemas de producción disponen de la infraestructura básica para la cría de ganado en cuanto a los comederos y bebederos y mangas; menos frecuente es la existencia de salas de ordeño y de establos para criar terneros, lo cual se presenta en porcentajes no altos en las UPA's de mayor superficie y que tienen mayor cantidad de ganado. El uso y disponibilidad de la cerca eléctrica es más común en la RMH y RSH$\mathrm{TE}$, no así en las otras regiones, lo cual dificulta el manejo adecuado de los pastizales de manera que se pueda suministrar la cantidad adecuada de pasto así como la calidad de la pastura controlando la rotación de potreros principalmente (ver Figura 8).

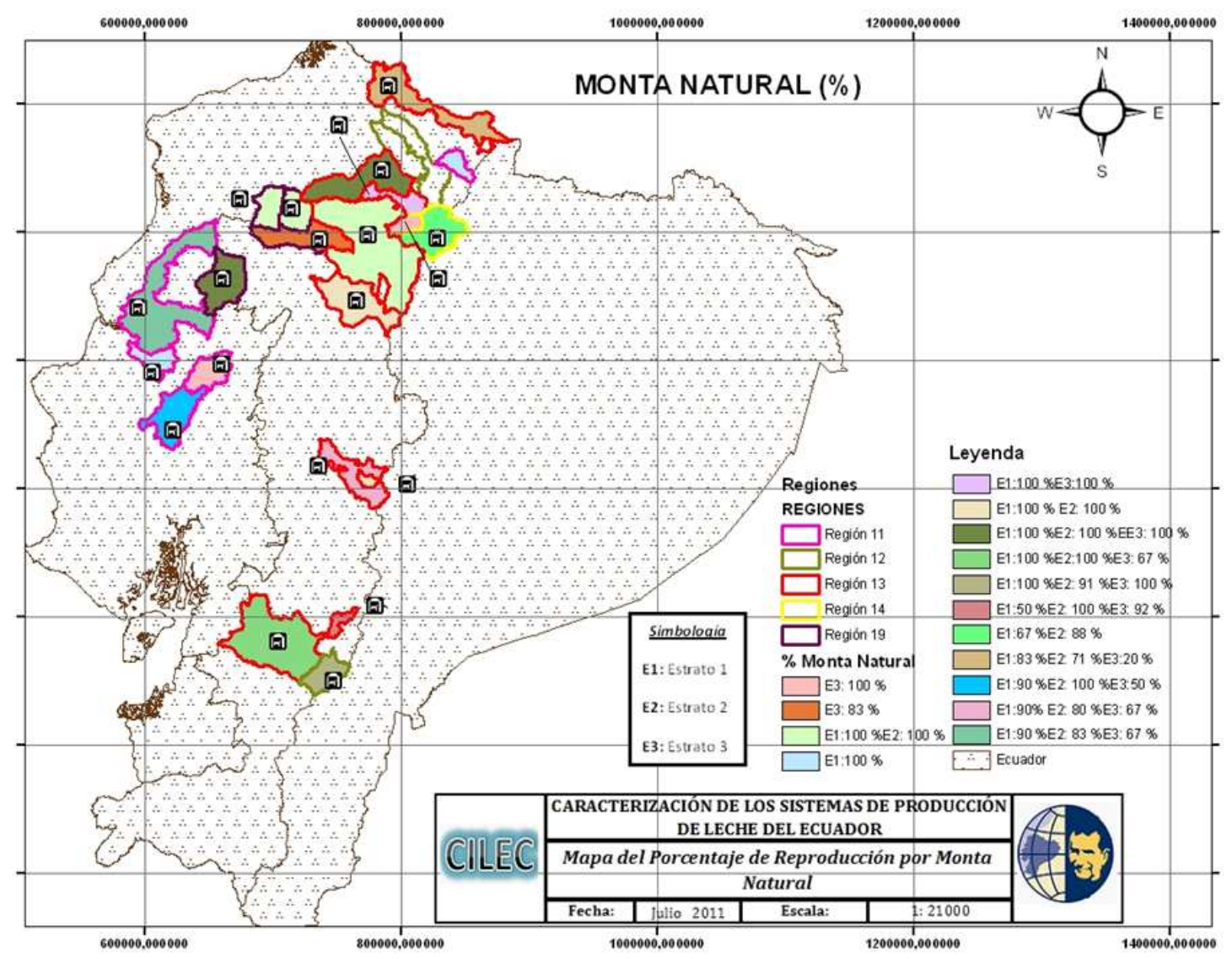

Figura 7. Forma de reproducción por estrato y por regiones

Otros equipos importantes en la actividad ganadera son los tanques de frio que se dispone para preservar la calidad higiénica de la leche; en los cantones de la Costa no es frecuente este equipamiento de manera individual, existiendo centros de acopio a veces particulares que acopian y enfrían la leche; en los cantones de la Sierra, este equipamiento exis- te en mayor cantidad y se pueden constatar centros de acopio comunitarios con tanques de frio que acopian y enfrían la leche, sin embargo, en los cantones del austro principalmente y del noroccidente de la provincia de Pichincha es limitada la existencia de este equipamiento (Figura 9). 


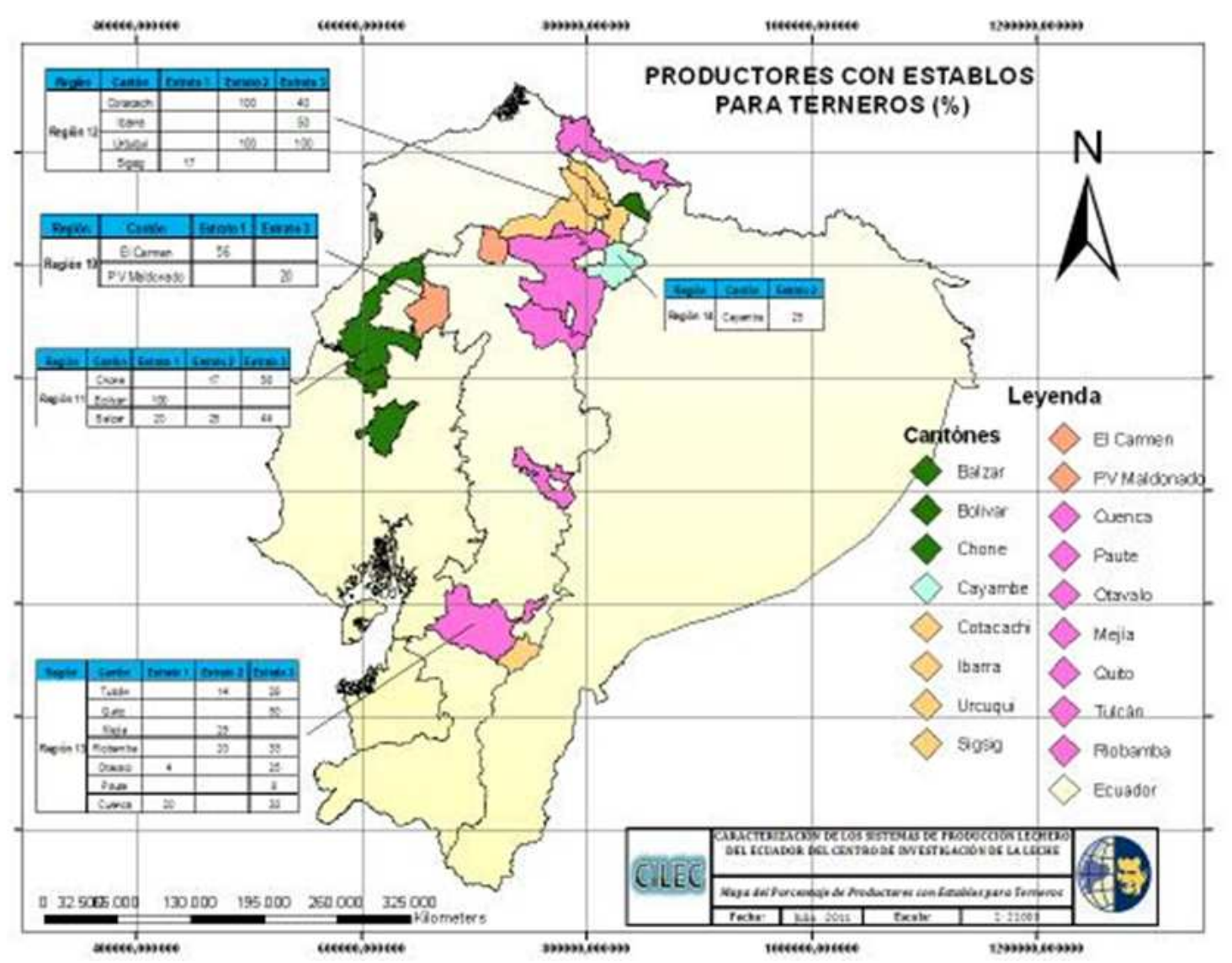

Figura 8. Productores que disponen de establos para terneros por regiones.

\section{Conclusiones y recomendacio- nes}

La lechería en Ecuador en los últimos años se ha desarrollado en función de la incorporación de superficie, pero, tal como hemos visto en los datos de la investigación es imprescindible definir políticas dirigidas a los pequeños productores (de 1 a 5 ha en la Sierra y $<20$ ha en la Costa) quienes con su mano de obra subvencionan la actividad ganadera.

Si bien es cierto que los SPL, por las características de las zonas agroecológicas, se desarrollan sin mayores inversiones, estos necesitan ser potencializados a través del uso adecuado de los recursos, especialmente el suelo y agua, de manera que se asegure la provisión de alimento para el ganado, esto pasa también por dotar de infraestructura de riego por aspersión a las fincas dedicadas a la ganadería, investigar sobre la fertilidad e hidromorfismo del suelo, así como del rendimiento de los pastizales y valor nutritivo de especies forrajeras nativas a fin de trabajar balances forrajeros que mantengan niveles de producción y reproducción óptimos y apunten a la sostenibilidad de la actividad ganadera.

\section{Agradecimientos}

A la Universidad Politécnica Salesiana por propiciar la generación de información y conocimiento sobre la situación de uno de los importantes sectores productivos del país como es el pecuario, a la Universidad Politécnica Estatal del Carchi, Pasteurizadora Carchi, Toni, Nutrileche, Improlac y por supuesto a nuestros estudiantes y a los productores de las provincias investigadas por su gentil colaboración. 


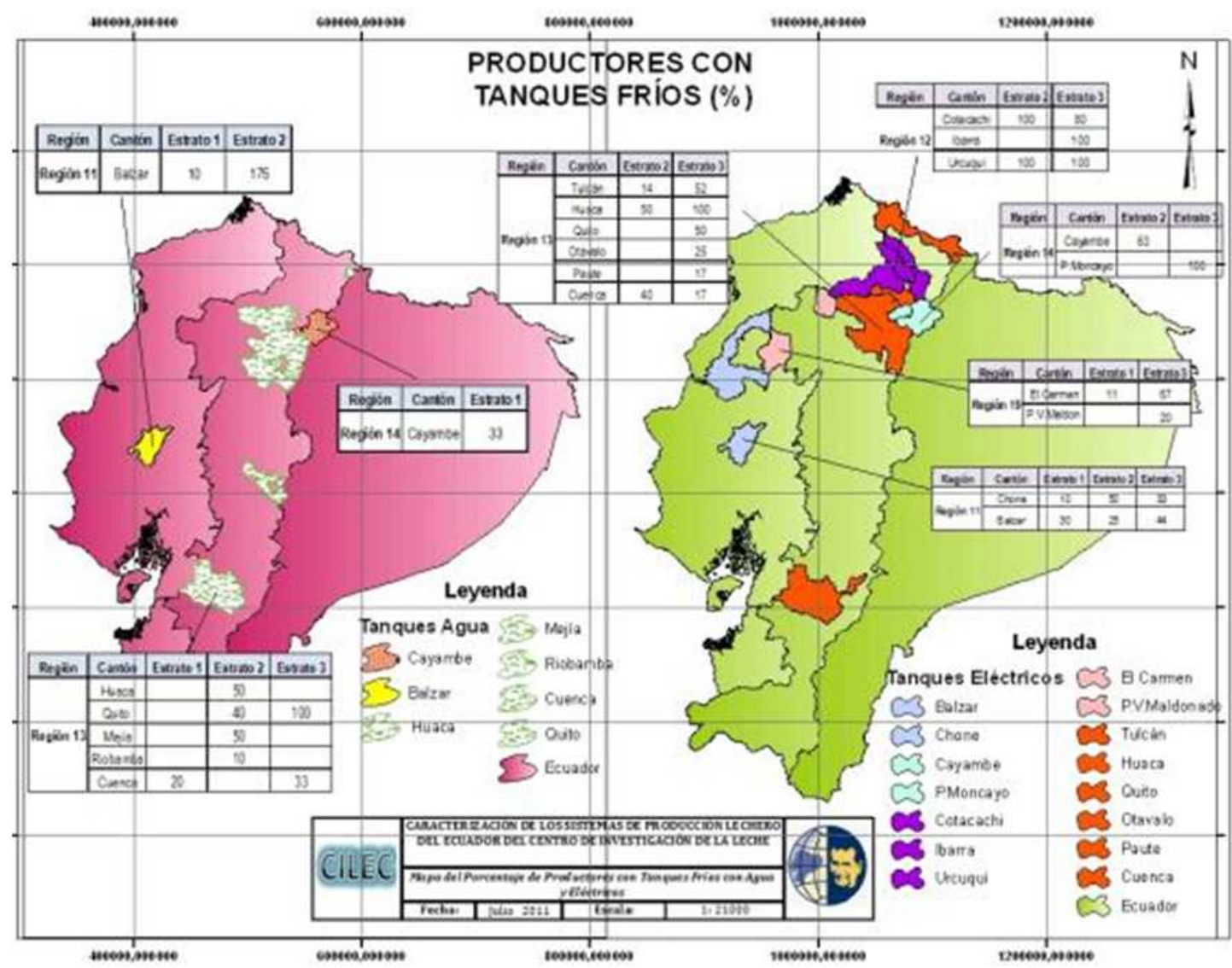

Figura 9. Productores que disponen de tanques para enfriamiento de leche por Regiones.

\section{Referencias}

Amaral, N. 2001. La competitividad de la cadena de lácteos en Mercosur. Instituto interamericano de Cooperación para la Agricultura Jhon Dixon y Aidan Gulliver, et. Al. Sistemas de producción agropecuaria y pobreza. FAO.

Barrionuevo, V. H. 1990. Evaluación del pasto estrella (cynodon nlenfuensis) solo y asociado con las leguminosas forrajeras arachis pintoi ciat 17434 y desmodium oxalifolium ciat $\mathbf{3 5 0}$ en la producción de leche y sus componentes. Proyecto Fin de Carrera, Costa Rica.

CentreI, I. D. R. Investigación en sistemas de producción pecuaria: 10 años de experiencia en México y Centroamérica. URL 〈http://web.idrc.

Cruz, L. C. 1983. El Mapa bioclimático y ecológico del Ecuador. MAG-PRONAREG.
García, N. B. y N. J. Requelme. 2011. Buenas prácticas de ordeño y la calidad higiénica de la leche en el ecuador. La Granja, 14(2): 45-57.

Laki, P. 1995. De la dependencia al protagonismo del agricultor. FAO. Santiago de Chile. 1995. FAO.

Larrea, T. 2011. Caracterización y eficiencia de la producción lechera en el noreste de la pampa argentina. Tesis Doctoral, Universidad de Córdova España.

MAGAP. 2011. Censo nacional agropecuario. Informe técnico.

MAGAP-GTZ. 1994. Proyecto de Fomento Ganadero.

Paladines, O. M. 1992. Metodología de pastizales para trabajar en fincas y proyectos de desarrollo agropecuario. PROFOGAN-MAG-GTZ. 
PROFOGAN. 1994. Proceso de análisis y mejoramiento de sistemas de producción agropecuario - forestales de pequeños y mediano productores. Experiencias del PRFOGAN en una zona baja y seca del callejón interandino.

Proyecto SICA. 2010. Cámara de agricultura de la primera zona. análisis e interpretación del iii censo agropecuario. Informe técnico, URL 〈http:/ / www.agroecuador.com/HTML/Censo /Censo.htm $\rangle$.

Ramirez, N., P. Izquierdo y C. Freddy. 1996. Producción y utilización de pastizales en cinco zo- nas agroecológicas del Ecuador. MAG- GTA- REPAAN.

Ramírez, P. y F. Izquierdo. 1996. Producción y utilización de pastizales en cinco zonas agroecológicas del país. MAG-GTZ-REPAAN.

Spedding. 1979. Organización de las Naciones Unidas para la Agricultura y la Alimentación. Roma. Análisis de sistemas de producción animal. Tomo 1: Las bases conceptuales.

Velarde, C. L. y I. C. Freddy. 1993. Producción y utilización de los pastizales de la zona altoandina. Red de Pastizales Andinos- REPAAN. 